\title{
Cosmological Structure Problem of the Pre-Big Bang Scenario
}

\author{
Jai-chan Hwang \\ Department of Astronomy and Atmospheric Sciences \\ Kyungpook National University, Taegu, Korea
}

October 27, 2018

\begin{abstract}
We calculate the density and gravitational wave spectrums generated in a version of string cosmology termed pre-big bang scenario. The large scale structures are originated from quantum fluctuations of the metric and dilaton field during a pole-like inflation stage driven by a potential-less dilaton field realized in the low-energy effective action of string theory. The generated classical density field and the gravitational wave in the second horizon crossing epoch show tilted spectrums with $n \simeq 4$ and $n_{T} \simeq 3$, respectively. These differ from the observed spectrum of the large angular scale anisotropy of the cosmic microwave background radiation which supports scale invariant ones with $n \simeq 1$ and $n_{T} \simeq 0$. This suggests that the pre-big bang stage is not suitable for generating the present day observable large scale structures, and suggests the importance of investigating the quantum generation processes during stringy era with higher order quantum correction terms.
\end{abstract}

PACS numbers: 04.30.-w, 04.50.+h, 98.80.Cq

Keywords: cosmology, string theory, large scale structure in the universe, gravitational wave

\section{Introduction}

String theory has attracted much attention in the literature as a successful candidate for unifying gravity with other fundamental interactions in the nature as a consistent theory of quantum gravity, [1]. However, effects of the string theory become significant on scales far beyond the range of direct experimental test. Theoretical successes provided by introducing an acceleration stage in the early universe in solving the structure generation processes in causal manner and other cosmological problems made the inflationary idea settled as an orthodox paradigm in the cosmology community, [2]. From the observational side we have some important clues which provide constraints on theories of the early universe (thus, theories of the high energy interactions) for the successful cosmology. The most stringent constraint for filtering the viable theory is provided by the large angular scale anisotropy of the cosmic microwave background radiation (CMBR). Both the amplitude and fluctuation spectrum of the microwave photons can be used as the primary filters.

Combining above three pieces of ideas (string theories, inflation models, and cosmological observations) we can think of the following. If we can make inflationary models based on string theories, the theories can be put under the testing ground of the cosmological observations. There were some attempts to find viable inflationary models based on the low energy effective action of string theories. One successful candinate is the one introduced in [3] termed "pre-big bang scenario" which generically predicts a pole-like inflation stage, [3]. In this paper, we will calculate the generated density and gravitational wave spectrums and the consequent anisotropy in the CMBR, thus situating the theory under the screening process of cosmological observations.

Recently, we have presented a unified formulation of treating the cosmological scalar type perturbations in generalized gravity theories, [4, 5, 6, 7]. Compared with previous work on the subject, a progress was made by discovering a proper choice of the gauge which suits the problem. The uniform-curvature gauge was introduced in [8] as one of several fundamental gauge choices available in treating the cosmological perturbations. Classical evolution stage is studied in 田, 5, 60]: Under the proper choice of the gauge (equivalently, gauge invariant variables) the behavior in the large scale is characterized by a conserved quantity. Ignoring the transient mode, the solution known in a minimally coupled scalar field 
remains valid in the generalized gravity theories. Quantum generation stage is studied in [7]: Using the uniform-curvature gauge, the quantum fluctuations generated in generic phases of acceleration stage can be calculated in analytic forms. The gravitational wave counterpart of the unified formulation (both for handling the classical evolution and the quantum generation processes) is recently presented in [9].

In the point of view of our generalized gravity theories considered in [4, [7, 9] the low energy effective action of string theory can be regarded as a simple subset. Thus, we will present the structure generation and evolution processes in the string theory by reducing the general results in [4, 6, 9]. Our study presented below will be based on the original frame of the string theory.

\section{Cosmological setting for string theory}

The low-energy effective action of string theory is [10]

$$
S=\int d^{4} x \sqrt{-g} \frac{1}{2} e^{-\phi}\left(R+\phi^{; a} \phi_{, a}\right) .
$$

The gravitational field equation and the equation of motion become [eqs. $(2,3)$ of [⿴囗⿰丿㇄口]:

$$
R_{a b}=-\phi_{, a ; b}, \quad \square \phi=\phi^{; a} \phi_{, a} .
$$

As a cosmological model describing the universe with structures, we consider a homogeneous and isotropic (flat) background with the general scalar and tensor type perturbations as

$$
d s^{2}=-(1+2 \alpha) d t^{2}-\chi_{, \alpha} d t d x^{\alpha}+a^{2}\left[\delta_{\alpha \beta}(1+2 \varphi)+2 C_{\alpha \beta}^{(t)}\right] d x^{\alpha} d x^{\beta},
$$

where $\alpha(\mathbf{x}, t), \chi(\mathbf{x}, t)$ and $\varphi(\mathbf{x}, t)$ are the scalar type metric perturbations; $C_{\alpha \beta}^{(t)}(\mathbf{x}, t)$ is a tracefree-transverse

$\left(C_{\alpha}^{(t) \alpha}=0=C_{\alpha, \beta}^{(t) \beta}\right)$ tensor type perturbation corresponding to the gravitational wave. We have ignored the rotation mode in the metric whose behavior is trivially characterized by the angular momentum conservation [Sec. 3.2.2 of [11]]. We consider perturbations in the dilaton field as $\phi(\mathbf{x}, t)=\bar{\phi}(t)+\delta \phi(\mathbf{x}, t)$ where a background quantity is indicated by an overbar which will be neglected unless necessary. Equations describing the background become [eqs. (20-22) of 四]:

$$
H^{2}=H \dot{\phi}-\frac{1}{6} \dot{\phi}^{2}, \quad \ddot{\phi}+3 H \dot{\phi}-\dot{\phi}^{2}=0
$$

where $H \equiv \dot{a} / a$.

\section{Formulation}

In our previous work [4, 5, 6, 4, 9], we presented the classical evolution and the quantum generation processes (both for the scalar type perturbation and the gravitational wave) generally valid in a class of gravity theories represented by an action

$$
S=\int d^{4} x \sqrt{-g}\left[\frac{1}{2} f(\phi, R)-\frac{1}{2} \omega(\phi) \phi^{; a} \phi_{, a}-V(\phi)\right] .
$$

The string theory in eq. (11) can be considered as a subset of theories in eq. (5) with $f=e^{-\phi} R, \omega=-e^{-\phi}$, and $V=0$. In ths section we summarize the classical evolution and the quantum generation processes for handling the scalar type perturbation and the gravitational wave which are quite generally applicable to second-order gravity theories included in eq. (5).

\subsection{Scalar type perturbation}

By introducing a gauge invariant combination

$$
\delta \phi_{\varphi} \equiv \delta \phi-\frac{\dot{\phi}}{H} \varphi \equiv-\frac{\dot{\phi}}{H} \varphi_{\delta \phi},
$$


the second order perturbed action of the scalar type perturbation of eq. (5) becomes [eq. (29) of [6]]

$$
\delta^{2} S=\frac{1}{2} \int a^{3} Z\left\{\delta \dot{\phi}_{\varphi}^{2}-\frac{1}{a^{2}} \delta \phi_{\varphi}^{\mid \alpha} \delta \phi_{\varphi, \alpha}+\frac{1}{a^{3} Z} \frac{H}{\dot{\phi}}\left[a^{3} Z\left(\frac{\dot{\phi}}{H}\right)\right] \delta \phi_{\varphi}^{2}\right\} d t d^{3} x .
$$

The non-Einstein nature of the theory is present in a parameter $Z$ defined as

$$
Z(t) \equiv \frac{\omega+\frac{3 \dot{F}^{2}}{2 \dot{\phi}^{2} F}}{\left(1+\frac{\dot{F}}{2 H F}\right)^{2}}
$$

where $F \equiv d f /(d R)$. The equations for the background in eq. (四) do not have Einstein limit. However, for the perturbation part, by taking $Z=1$ the perturbed action in eq. (7) reduces to the one valid in Einstein gravity with a minimally coupled scalar field. The equation of motion of the perturbed dilaton field follows as

$$
\delta \ddot{\phi}_{\varphi}+\frac{\left(a^{3} Z\right)^{\cdot}}{a^{3} Z} \delta \dot{\phi}_{\varphi}-\left\{\frac{1}{a^{2}} \nabla^{2}+\frac{1}{a^{3} Z} \frac{H}{\dot{\phi}}\left[a^{3} Z\left(\frac{\dot{\phi}}{H}\right)\right]\right\} \delta \phi_{\varphi}=0 .
$$

The large and small scale asymptotic solutions are, respectively:

$$
\begin{aligned}
& \delta \phi_{\varphi}(\mathbf{x}, t)=-\frac{\dot{\phi}}{H} \varphi_{\delta \phi}(\mathbf{x}, t)=-\frac{\dot{\phi}}{H}\left[C(\mathbf{x})-D(\mathbf{x}) \int^{t} \frac{1}{a^{3} Z} \frac{H^{2}}{\dot{\phi}^{2}} d t\right], \\
& \delta \phi_{\varphi}(\mathbf{k}, \eta)=\frac{1}{a \sqrt{2 k}}\left[c_{1}(\mathbf{k}) e^{i k \eta}+c_{2}(\mathbf{k}) e^{-i k \eta}\right] \frac{1}{\sqrt{Z}}
\end{aligned}
$$

where $C(\mathbf{x})$ and $D(\mathbf{x})$ are integration constants of the growing and decaying mode, respectively; by relaxing the lower bound of the integration in $D(\mathbf{x})$ term we took into account of the possible mixing of the modes through transition among different background evolution phases. $D(\mathbf{x})$ term is higher order in the large scale expansion compared with the solutions in the other gauges; see $\S$ VIA of [5]. At this point $c_{1}(\mathbf{k})$ and $c_{2}(\mathbf{k})$ are arbitrary integration constants. We emphasize that the solutions in Eqs. (10,11) are valid considering general evolutions in $V(\phi), \omega(\phi)$ and $f(\phi, R)$ as long as the gravity theories belong to Eq. (5) and second-order. $C(\mathbf{x}), D(\mathbf{x}), c_{1}(\mathbf{k})$ and $c_{2}(\mathbf{k})$ are integration constants considering such evolutions.

When we have $z^{\prime \prime} / z=n / \eta^{2}$ with $n=$ constant, where $z(t) \equiv \frac{a \dot{\phi}}{H} \sqrt{Z}$, eq. (9) becomes a Bessel equation with a solution [eq. (18) of [7]]

$$
\delta \phi_{\varphi \mathbf{k}}(\eta)=\frac{\sqrt{\pi|\eta|}}{2 a}\left[c_{1}(\mathbf{k}) H_{\nu}^{(1)}(k|\eta|)+c_{2}(\mathbf{k}) H_{\nu}^{(2)}(k|\eta|)\right] \frac{1}{\sqrt{Z}}, \quad \nu \equiv \sqrt{n+\frac{1}{4}} .
$$

Considering $\delta \phi_{\varphi \mathbf{k}}(\eta)$ as a mode function of $\delta \hat{\phi}(\mathbf{x}, t)$ which is regarded as a quantum Heisenberg operator, the canonical quantization condition leads to the following normalization condition []

$$
\left|c_{2}(\mathbf{k})\right|^{2}-\left|c_{1}(\mathbf{k})\right|^{2}=1 .
$$

The quantization condition does not fix the mode function completely. Choices of $c_{1}(\mathbf{k})$ and $c_{2}(\mathbf{k})$ depend on the vacuum state. The positive frequency solution in the small scale limit corresponds to taking $c_{2}=1$ and $c_{1}=0$ which is the simplest choice; it is also the choice always considered in the literature. The power spectrum based on the vacuum expectation value becomes

$$
\mathcal{P}_{\delta \hat{\phi}_{\varphi}}(k, t)=\frac{k^{3}}{2 \pi^{2}}\left|\delta \phi_{\varphi \mathbf{k}}(t)\right|^{2} .
$$

\subsection{Gravitational wave}

The second order perturbed action for the gravitational wave part of eq. (5) becomes [9]

$$
\delta^{2} S_{g}=\frac{1}{2} \int a^{3} F\left(\dot{C}_{\beta}^{(t) \alpha} \dot{C}_{\alpha}^{(t) \beta}-\frac{1}{a^{2}} C_{\beta, \gamma}^{(t) \alpha} C_{\alpha}^{(t) \beta \mid \gamma}\right) d t d^{3} x .
$$


The equation of motion is

$$
\ddot{C}_{\alpha \beta}^{(t)}+\left(3 H+\frac{\dot{F}}{F}\right) \dot{C}_{\alpha \beta}^{(t)}-\frac{1}{a^{2}} \nabla^{2} C_{\alpha \beta}^{(t)}=0
$$

The large and small scale asymptotic solutions are, respectively:

$$
\begin{aligned}
& C_{\alpha \beta}^{(t)}(\mathbf{x}, t)=C_{\alpha \beta}(\mathbf{x})-D_{\alpha \beta}(\mathbf{x}) \int^{t} \frac{1}{a^{3} F} d t \\
& C_{\alpha \beta}^{(t)}(\mathbf{k}, \eta)=\frac{1}{a \sqrt{F}}\left[c_{1 \alpha \beta}(\mathbf{k}) e^{i k \eta}+c_{2 \alpha \beta}(\mathbf{k}) e^{-i k \eta}\right] .
\end{aligned}
$$

Similar comments below Eq. (11) also apply to Eqs. (17,18). In order to handle the quantum generation process we consider a Hilbert space operator $\hat{C}_{\alpha \beta}^{(t)}$ instead of the classical metric perturbation $C_{\alpha \beta}^{(t)}$. We write

$$
\hat{C}_{\alpha \beta}^{(t)}(\mathbf{x}, t) \equiv \int \frac{d^{3} k}{(2 \pi)^{3 / 2}}\left[\sum_{\ell} e^{i \mathbf{k} \cdot \mathbf{x}} \tilde{h}_{\ell \mathbf{k}}(t) \hat{a}_{\ell \mathbf{k}} e_{\alpha \beta}^{(\ell)}(\mathbf{k})+\text { h.c. }\right]
$$

where $\ell=+, \times ; e_{\alpha \beta}^{(\ell)}$ is bases of two $(+$ and $\times)$ polarization states and $\hat{a}_{\ell}$ is the creation and annihilation operators of the polarization states. By introducing

$$
\hat{h}_{\ell}(\mathbf{x}, t) \equiv \int \frac{d^{3} k}{(2 \pi)^{3 / 2}}\left[e^{i \mathbf{k} \cdot \mathbf{x}} \tilde{h}_{\ell \mathbf{k}}(t) \hat{a}_{\ell \mathbf{k}}+\text { h.c. }\right],
$$

eq. (15) can be written as [12]

$$
\delta^{2} S_{g}=\int a^{3} F \sum_{\ell}\left(\dot{\hat{h}}_{\ell}^{2}-\frac{1}{a^{2}} \hat{h}_{\ell}^{\mid \gamma} \hat{h}_{\ell, \gamma}\right) d t d^{3} x
$$

For $z_{g}^{\prime \prime} / z_{g}=n_{g} / \eta^{2}$ with $n_{g}=$ constant, where $z_{g}(t) \equiv a \sqrt{F}$, eq. (16) has an exact solution. In terms of the mode function we have

$$
\tilde{h}_{\ell \mathbf{k}}(\eta)=\frac{\sqrt{\pi|\eta|}}{2 a}\left[c_{\ell 1}(\mathbf{k}) H_{\nu_{g}}^{(1)}(k|\eta|)+c_{\ell 2}(\mathbf{k}) H_{\nu_{g}}^{(2)}(k|\eta|)\right] \sqrt{\frac{1}{2 F}}
$$

where according to the canonical quantization condition the coefficients $c_{\ell 1}(\mathbf{k})$ and $c_{\ell 2}(\mathbf{k})$ follow

$$
\left|c_{\ell 2}(\mathbf{k})\right|^{2}-\left|c_{\ell 1}(\mathbf{k})\right|^{2}=1
$$

The simplest vacuum choice corresponds to taking $c_{\ell 2}=1$ and $c_{\ell 1}=0$. The power spectrum of the Hilbert space graviational wave operator based on the vacuum expectation value becomes

$$
\mathcal{P}_{\hat{C}_{\alpha \beta}^{(t)}}(\mathbf{k}, t)=2 \sum_{\ell} \mathcal{P}_{\hat{h}_{\ell}}(\mathbf{k}, t)=2 \sum_{\ell} \frac{k^{3}}{2 \pi^{2}}\left|\tilde{h}_{\ell \mathbf{k}}(t)\right|^{2} .
$$

Previous studies on the evolution of the gravitational wave in some generalized gravity theories can be found in $13,11,8]$.

Using $\epsilon_{i}$ 's introduced in eq. (87) of [5] we have

$$
\frac{z_{g}^{\prime \prime}}{z_{g}}=a^{2} H^{2}\left(1+\epsilon_{3}\right)\left(2+\epsilon_{1}+\epsilon_{3}\right)+a^{2} H \dot{\epsilon}_{3}
$$

For $\dot{\epsilon}_{i}=0$ we have

$$
n=\frac{\left(1-\epsilon_{1}+\epsilon_{2}-\epsilon_{3}+\epsilon_{4}\right)\left(2+\epsilon_{2}-\epsilon_{3}+\epsilon_{4}\right)}{\left(1+\epsilon_{1}\right)^{2}}, \quad n_{g}=\frac{\left(1+\epsilon_{3}\right)\left(2+\epsilon_{1}+\epsilon_{3}\right)}{\left(1+\epsilon_{1}\right)^{2}} .
$$




\section{Vacuum Fluctuations in the Pre-Big bang Scenario}

In the low energy effective action of the string theories, we have

$$
F=e^{-\phi}, \quad Z=\frac{1}{2} e^{-\phi}\left(1-\frac{\dot{\phi}}{2 H}\right)^{-2} .
$$

From eq. (87) of [5] we have:

$$
\epsilon_{1} \equiv \frac{\dot{H}}{H^{2}}, \quad \epsilon_{2} \equiv \frac{\ddot{\phi}}{H \dot{\phi}}, \quad 2 \epsilon_{3}=\epsilon_{4}=-\frac{\dot{\phi}}{H} .
$$

Equation (凷) has the following solution

$$
a_{\mp} \propto\left|t-t_{0}\right|^{\mp 1 / \sqrt{3}}, \quad e^{\phi_{\mp}} \propto\left|t-t_{0}\right|^{-1 \mp \sqrt{3}} .
$$

The $a_{-}$branch with $t<t_{0}$ represents a pole-like inflation stage which is called a pre-big bang stage [3]. We will consider this case in the following. We have

$$
\eta=-\frac{3-\sqrt{3}}{2} \frac{t_{0}-t}{a}, \quad \frac{\dot{\phi}}{H}=3+\sqrt{3}, \quad \delta \phi_{\varphi}=-(3+\sqrt{3}) \varphi_{\delta \phi}, \quad Z=(2-\sqrt{3}) e^{-\phi} .
$$

For the scalar type perturbation eqs. (7.9.10) become

$$
\begin{aligned}
& \delta^{2} S=\frac{1}{2} \int a^{3} Z\left(\delta \dot{\phi}_{\varphi}^{2}-\frac{1}{a^{2}} \delta \phi_{\varphi}^{\mid \alpha} \delta \phi_{\varphi, \alpha}\right) d t d^{3} x, \\
& \delta \ddot{\phi}_{\varphi}-\frac{1}{t_{0}-t} \delta \dot{\phi}_{\varphi}-\frac{1}{a^{2}} \nabla^{2} \delta \phi_{\varphi}=0 \\
& \delta \phi_{\varphi}(\mathbf{x}, t)=-(3+\sqrt{3})\left[C(\mathbf{x})+\frac{D(\mathbf{x})}{3(3+\sqrt{3})}\left(\frac{|\eta|}{a^{2} Z}\right)_{1} \ln \left(1-t / t_{0}\right)\right] .
\end{aligned}
$$

Since $|\eta| /\left(a^{2} Z\right)=$ constant we evaluated it at an arbitrary epoch $t_{1} . D(\mathbf{x})$ term in Eq. (33) logarithmically diverges near the end of the pre-big bang phase as $t$ approaches $t_{0}$. Equation (28) becomes $\epsilon_{1}=\epsilon_{2}=\sqrt{3}$, $2 \epsilon_{3}=\epsilon_{4}=-3-\sqrt{3}$. From eq. (26) we have $n=-1 / 4$, thus $\nu=0$. Thus, eq. (12) becomes

$$
\delta \phi_{\varphi \mathbf{k}}(\eta)=\frac{\sqrt{\pi}}{2}\left(\sqrt{\frac{|\eta|}{a^{2} Z}}\right)_{1}\left[c_{1}(\mathbf{k}) H_{0}^{(1)}(k|\eta|)+c_{2}(\mathbf{k}) H_{0}^{(2)}(k|\eta|)\right] .
$$

The general power spectrum can be found from eqs. (14,34). In the large scale limit we have

$$
\begin{aligned}
\mathcal{P}_{\delta \hat{\phi}_{\varphi}}^{1 / 2}(\mathbf{k}, \eta) & =(3+\sqrt{3}) \mathcal{P}_{\hat{\varphi}_{\delta \phi}}^{1 / 2}(\mathbf{k}, \eta) \\
& =\frac{3+\sqrt{3}}{\sqrt{6}}\left[\frac{H}{\sqrt{Z}}\left(\frac{k|\eta|}{\pi}\right)^{3 / 2}\right]_{1}|\ln (k|\eta|)| \times\left|c_{2}(\mathbf{k})-c_{1}(\mathbf{k})\right|
\end{aligned}
$$

where we used $\eta=-\frac{\sqrt{3}-1}{2} \frac{1}{a H}$. Authors of 14] derived the power spectrum of quantum fluctuations in a string era in a context of conformally related Einstein frame assuming the simplest vacuum choice.

For the gravitational wave we can apply the formulation in 83.2 with $F=e^{-\phi}$. From eq. (26) we have $n_{g}=-\frac{1}{4}$, thus $\nu_{g}=0$. The general power spectrum follows from eqs. (22,24). In the large scale limit we have

$$
\mathcal{P}_{\hat{C}_{\alpha \beta}^{(t)}}^{1 / 2}(\mathbf{k}, \eta)=\sqrt{2}\left[\frac{H}{\sqrt{Z}}\left(\frac{k|\eta|}{\pi}\right)^{3 / 2}\right]_{1}|\ln (k|\eta|)| \times \sqrt{\frac{1}{2} \sum_{\ell}\left|c_{\ell 2}(\mathbf{k})-c_{\ell 1}(\mathbf{k})\right|^{2}} .
$$

The gravitational wave power spectrum in the pre-big bang scenario has been studied in [15, 14 assuming the simplest vacuum choice.

By comparing eqs. (35, 36), and taking the simplest vacuum choices, we have

$$
\mathcal{P}_{\hat{C}_{\alpha \beta}^{(t)}}^{1 / 2}(\mathbf{k}, t)=2 \sqrt{3} \times \mathcal{P}_{\hat{\varphi}_{\delta \phi}}^{1 / 2}(\mathbf{k}, t) .
$$




\section{Generated Classical Spectrums}

In the large scale limit, ignoring the decaying mode, from eq. (10) we have

$$
\mathcal{P}_{C}^{1 / 2}(\mathbf{k})=\mathcal{P}_{\varphi_{\delta \phi}}^{1 / 2}(\mathbf{k}, t) \equiv \mathcal{P}_{\hat{\varphi} \delta \phi}^{1 / 2}(\mathbf{k}, t) \times Q^{1 / 2}(\mathbf{k})
$$

where $\mathcal{P}_{f}$ and $\mathcal{P}_{\hat{f}}$ are power spectrums based on the space averaging of the classically fluctuating field $f(\mathbf{x}, t)$ and on the vacuum expectation value of the fluctuating quantum operator $\hat{f}(\mathbf{x}, t)$, respectively. $Q(k)$ is a classicalization factor which may take into account of possible effects from the classicalization processes [16. The second step in eq. (38) should be considered as an ansatz. Similarly, for the gravitational wave we take the following ansatz: in the large scale limit during inflation era we assume

$$
\mathcal{P}_{C_{\alpha \beta}}(\mathbf{k})=\mathcal{P}_{C_{\alpha \beta}^{(t)}}(\mathbf{k}, \eta)=2 \sum_{\ell} \mathcal{P}_{h_{\ell}}(\mathbf{k}, \eta) \equiv 2 \sum_{\ell} \mathcal{P}_{\hat{h}_{\ell}}(\mathbf{k}, \eta) \times Q_{\ell}(\mathbf{k}),
$$

where $Q_{\ell}(\mathbf{k})$ is a classicalization factor for the gravitational wave with a polarization state $\ell$.

Thus, finally, from eqs. (38, 35) and eqs. (39, 36) we have

$$
\begin{aligned}
\mathcal{P}_{C}^{1 / 2}(\mathbf{k})=\mathcal{P}_{\varphi_{\delta \phi}}^{1 / 2}(\mathbf{k}, \eta)=\frac{1}{\sqrt{6}} & {\left[\frac{H}{\sqrt{Z}}\left(\frac{k|\eta|}{\pi}\right)^{3 / 2}\right]_{1}|\ln (k|\eta|)| \times\left|c_{2}(\mathbf{k})-c_{1}(\mathbf{k})\right| \sqrt{Q(k)}, } \\
\mathcal{P}_{C_{\alpha \beta}}^{1 / 2}(\mathbf{k})=\mathcal{P}_{C_{\alpha \beta}^{(t)}}^{1 / 2}(\mathbf{k}, \eta)=\sqrt{2} & {\left[\frac{H}{\sqrt{Z}}\left(\frac{k|\eta|}{\pi}\right)^{3 / 2}\right]_{1}|\ln (k|\eta|)| } \\
& \times \sqrt{\frac{1}{2} \sum_{\ell}\left|c_{\ell 2}(\mathbf{k})-c_{\ell 1}(\mathbf{k})\right|^{2} \times Q_{\ell}(\mathbf{k}),}
\end{aligned}
$$

where the right hand sides should be evaluated while the scale stays in the large scale during the pre-big bang era. [The logarithmic time dependent terms in eqs. (40,41) look inconsistent because $C$ and $C_{\alpha \beta}$ are time independent. We guess this point occurs because we have considered the quantum fluctuations in the full field $\varphi_{\delta \phi}$ contributing to the pure growing mode characterized by $C(\mathbf{x})$, and similarly for the gravitational wave.] By comparing eqs. (40,41), taking the simplest vacuum choices and ignoring the classicalization factors, we have

$$
\mathcal{P}_{C_{\alpha \beta}^{(t)}}^{1 / 2}(\mathbf{k}, \eta)=2 \sqrt{3} \times \mathcal{P}_{\varphi_{\delta \phi}}^{1 / 2}(\mathbf{k}, \eta) .
$$

Up to this point we have calculated the quantum fluctuations which are pushed outside the horizon, classicalized, and imprinted in the spatial coeffiecients of the classical solutions in eqs. (10,17). These solutions characterize the large scale evolutions. Now, we would like to argue that the large scale evolution of the perturbations is characterized by conserved quantities independently of the changes in the background equation of state (including the transition from the string dominated era to the ordinary radiation or matter dominated universe), which can affect physics in the subhorizon scale. The scenario we have in mind is the one in which the observationally relevant fluctuations become superhorizon scale (thus, large scale) during the acceleration stage and come back into the subhorizon scale during the ordinary matter dominated era effectively governed by Einstein's gravity. From eqs. (10,17), ignoring the decaying modes which is higher order for $\varphi_{\delta \phi}$, we have

$$
\varphi_{\delta \phi}(\mathbf{x}, t)=C(\mathbf{x}), \quad C_{\alpha \beta}^{(t)}(\mathbf{x}, t)=C_{\alpha \beta}(\mathbf{x}) .
$$

Thus, in large scale limit the growing modes of $\varphi_{\delta \phi}$ and $C_{\alpha \beta}^{(t)}$ are conserved. We note that this conserved behavior is generally valid for a class of generalized gravity which includes our string theory; i.e., considering general $V(\phi), \omega(\phi)$, and $f(\phi, R)$ in eq. (5). Let us make this point more clear: As long as the two conditions [first, the transition process involves only the gravity theories which are subsets of Eq. (5), and second, the scale remains in the large scale during the transition] are held, we have a general large scale solution in Eq. (10) which is

$$
\varphi_{\delta \phi}(\mathbf{x}, t)=C(\mathbf{x})-D(\mathbf{x}) \int^{t} \frac{1}{a^{3} Z} \frac{H^{2}}{\dot{\phi}^{2}} d t
$$


Remarkably, this equation (and Eq. [17]) is valid considering general evolutions of $V(\phi), f(\phi, R), \omega(\phi)$ etc., as long as the evolution occurs satisfying the two conditions. As mentioned before, $C(\mathbf{x})$ and $D(\mathbf{x})$ are "integration constants" considering the general evolutions. Thus, as long as the two conditions are met, Eq. (44) takes care of the general evolution from the pre-big bang phase to Einstein gravity, and although there can arise the mixing in the $D(\mathbf{x})$ mode, the growing $C(\mathbf{x})$ will not be affected. This argument justify ignoring the decaying mode in the large scale evolution; the growing $C(\mathbf{x})$ mode in Einstein phase is only affected by the same $C(\mathbf{x})$ mode in the pre-big bang phase.

This conservation in the large scale may reflect the kinematic nature of the evolution in the superhorizon scale. Equation (43) is valid in a scale larger than the sound horizon for the scalar type perturbation and the visual horizon for the gravitational wave. Notice that the generalized nature of the gravity theory, $Z$ or $F$, does not appear in eq. (43). In Einstein's gravity with the ordinary fluid and the general equation of state, i.e., $p=p(\mu)$, we have the same conserved behavior again as in eq. (43), but now $\varphi_{\delta \phi}$ being replaced by $\varphi_{\Psi}$, where $\Psi=0$ corresponds to taking the comoving gauge, [8]. [In Einstein gravity the uniform-field gauge is the same as the comoving gauge with $\Psi=-\dot{\phi} \delta \phi$, 17]. For thorough discussions concerning the large scale conserved quantities in the fluid era and the minimally coupled scalar field, see [18.]

Armed with this knowledge from previous studies, we can understand the classical evolution of the perturbations in the large scale in the following manner: In addition to the quantum fluctuation of the tranverse-tracefree part of the metric, the quantum fluctuation in the dilaton field simultaneously excites and accompanies quantum fluctuations in the scalar type metric. During the acceleration era the relevant scale becomes superhorizon size and becomes classical. While the perturbations are superhorizon size they can be characterized by the conserved quantities $C(\mathbf{x})$ and $C_{\alpha \beta}(\mathbf{x})$ which are in fact $\varphi_{\delta \phi}$ and $C_{\alpha \beta}^{(t)}$, respectively. [For handling the gravitational wave the often favored method in the literature is matching the Bogoliubov coefficients assuming sudden jump transitions among different background expansion stages [19]. In this paper we use the general conservation property of the gravitational wave in the large scale; this method was originally used in [20].] We do not imply that everything is conserved, but rather imply that, from the conserved quantity the behavior of every other variable follows as linear combination. In the linear theory, all variables are linearly related with each other. From any one known solution we can derive all the other. Thus, we can regard the informations of the spatial structures are encoded in $C(\mathbf{x})$ and $C_{\alpha \beta}(\mathbf{x})$, and this informations are preserved as long as the scale remains in the large scale (Jeans scale in the fluid era for the scalar type perturbation). In this sense, changing phases of the underlying background universe including the gravity sector and the equation of state do not affect the evolution of structures in the superhorizon scale. [This is true, at least, as long as the gravity is included in eq. (5). We expect (but yet to be proved!) it to be more generally true reflecting the kinematic nature of physics in the superhorizon scale.] Thus, as long as the graceful exit problem in the pre-big bang scenario can be settled with reasonable modifications of the gravity sector, our description of the superhorizon size perturbations in terms of a conserved quantity is expected to remain valid; for the graceful exit problem in the pre-big bang scenario, see 21, 22]. As the scale comes back inside horizon, informations about observationally relevant quantities can be decoded from the conserved quantities $C(\mathbf{x})$ and $C_{\alpha \beta}(\mathbf{x})$.

In the matter dominated era, the solution for the relative density fluctuation $(\delta \equiv \delta \rho / \rho)$ becomes [eq. (23) of [4]

$$
\delta(\mathbf{x}, t)=\frac{2}{5}\left(\frac{k}{a H}\right)^{2} C(\mathbf{x})
$$

Thus, in the second horizon crossing, where $\left.\frac{k}{a H}\right|_{\mathrm{HC}} \equiv 1$, we have

$$
\mathcal{P}_{\delta}^{1 / 2}\left(k, t_{\mathrm{HC}}\right)=\frac{2}{5} \mathcal{P}_{C}^{1 / 2}(k)=\frac{(a H)^{2}}{\sqrt{2} \pi} k^{-1 / 2}\left|\delta_{\mathbf{k}}(t)\right| .
$$

Conventionally we take $\left|\delta_{\mathbf{k}}(t)\right|^{2} \equiv A(t) k^{n}$ where $n$ is a spectral index; $n=1$ corresponds to the Zeldovich spectrum. Ignoring the classicalization factor $(Q \equiv 1)$, the vacuum dependence $\left(c_{2} \equiv 1\right.$ and $\left.c_{1} \equiv 0\right)$, and the logarithmic dependence on $k$, from eqs. (40,46) we have a tilted spectrum with $n=4$.

Similarly for the gravitational wave, in the matter dominated era and ignoring the transient mode, 
we conventionally take

$$
\mathcal{P}_{C_{\alpha \beta}^{(t)}}^{1 / 2}(\mathbf{k}, t)=\frac{\sqrt{2}}{\pi} A_{T}^{1 / 2}(\mathbf{k})\left|\frac{3 j_{1}(k \eta)}{k \eta}\right| .
$$

By matching eq. (41) with eq. (47) in the large scale limit we can show that

$$
A_{T}^{1 / 2}(\mathbf{k})=\pi\left[\frac{H}{\sqrt{Z}}\left(\frac{k|\eta|}{\pi}\right)^{3 / 2}\right]_{1}|\ln (k|\eta|)| \times \sqrt{\frac{1}{2} \sum_{\ell}\left|c_{\ell 2}(\mathbf{k})-c_{\ell 1}(\mathbf{k})\right|^{2} \times Q_{\ell}(\mathbf{k})} .
$$

One often writes $A_{T}(\mathbf{k}) \equiv A_{T} k^{n_{T}}$. If we ignore the dependences on the choice of the vacuum state and the classicalization factor, we have $n_{T}=3$. This spectrum also differs from the scale invariant one with $n_{T}=0$. The gravitational waves generated in the pre-big bang scenarios are thoroughly investigated in 115, 14.

The CMBR last scattered in the recombination era must have gone through the perturbed spacetime. The spatially and temporally fluctuating spacetime metric causes the directional (e) dependence of the CMBR temperature observed in a given position $\mathbf{x}_{R}$ as $\delta T\left(\mathbf{e} ; \mathbf{x}_{R}\right)$, 23. Conventionally we expand $\frac{\delta T}{T}\left(\mathbf{e} ; \mathbf{x}_{R}\right)=\sum_{l m} a_{l m}\left(\mathbf{x}_{R}\right) Y_{l m}(\mathbf{e})$. We are often interested in the rotationally symmetric quantity

$\left\langle a_{l}^{2}\right\rangle \equiv\left\langle\left|a_{l m}\left(\mathbf{x}_{R}\right)\right|^{2}\right\rangle_{\mathbf{x}_{R}}$ where \langle\rangle$_{\mathbf{x}_{R}}$ is an average over possible locations of an observer. In the following we present the result in order to show the proper normalization with our notation. For the scalar type perturbation and the gravitational wave, we have, respectively [24]

$$
\begin{aligned}
& \left\langle a_{l}^{2}\right\rangle=\frac{4 \pi}{25} \int_{0}^{\infty} \mathcal{P}_{C}(k) j_{l}^{2}(k x) d \ln k, \quad\left\langle a_{l}^{2}\right\rangle=\frac{9 \pi}{2} \frac{\Gamma(l+3)}{\Gamma(l-1)} \int_{0}^{\infty} A_{T}(k)\left|I_{l}(k)\right|^{2} d \ln k, \\
& I_{l}(k) \equiv \frac{2}{\pi} \int_{\eta_{e}}^{\eta_{o}} \frac{j_{2}(k \eta)}{k \eta} \frac{j_{l}\left(k \eta_{0}-k \eta\right)}{\left(k \eta_{0}-k \eta\right)^{2}} k d \eta,
\end{aligned}
$$

where $x=2 / H_{0}$. By integrating eq. (49) using eqs. (40,48) we can estimate the effects on the CMBR. The observational results favor $n \simeq 1$ and $n_{T} \simeq 0$, 25.

\section{Comparison with previous works and Discussions}

Studies of the scalar type fluctuations in pre-big bang scenario were also carried out in [26, 14]. The study in 26] is based on a zero-shear gauge which complicates the analysis compared with the one based on the uniform-curvature gauge used in our approach. Authors in [14] introduced an off-diagonal gauge which is in fact the same as the uniform-curvature gauge. Studies in 22, 14 are made in a conformally related Einstein frame. In the classical perturbation level the effective string theory in eq. (1) and Einstein's gravity can be transformed to each other through the conformal rescaling; this is shown in more general context of eq. (5) in [6]. The equivalence in the fully quantum level is not obvious, [27]. However, in the quantum level of our perturbative semiclassical approximation, treating both the perturbed dilaton field and the perturbed metric as quantum operators, we can show the equivalence through the conformal transformation, see [7. Our study presented above is directly made in the original frame of the string theory both for the quantum generation and the classical evolution; we may stress that it is hardly more difficult than working in Einstein frame. Authors of 14. derived only the power spectrum of the quantum fluctuations in the string era which corresponds to eq. (35). We have been able to derive the final density spectrum at the second horizon epoch and present day universe (thus partly complete the structure formation) by assuming a transition of the underlying gravity, from the string theory to the Einstein one, while the observationally relevant perturbations were on superhorizon scale. The transition of the string stage into Einstein one is a serious assumption which needs further investigation; though, we made an argument that as long as the perturbations we are interested in are in the superhorizon scale and as long as the gravity belongs to Eq. (5), we can apply the conservation argument of the perturbed curvature variable in the uniform field (or comoving) gauge as in eq. (43); see below Eq. (42). Equations (45.49) are valid if the present day universe is governed by the Einstein gravity.

In this paper we have not estimated the realistic amplitude of the observationally relevant quantities which may depend on the specific realization of the pre-big bang scenario. However, the power spectrums 
with $n \simeq 4$ and $n_{T} \simeq 3$ in eqs. (40 41 are in contrast with the observationally supported spectrum with $n \simeq 1$ and $n_{T} \simeq 0$, 225. This implies that the spectrum generated in the above theory cannot be considered as the source for the observed fluctuations in the CMBR and also the observed large scale structures. At this point, we would like to point out the possibility that the choices of the vacuum states and the classicalization factors in eqs. (40,41) can completely dominate the whole power spectrum. Effects of these two factors cannot be estimated within the scope of our linear treatment of the quantum fluctuations, and may require other physical arguments.

In $\oint$ we have derived the final observational results based on a couple of important assumptions: The first assumption is that the observationally relevant scale exits the horizon to become a superhorizon size, and becomes classical during the pre-big bang era. The second assumption is that at some epoch in the early universe the gravity transits from the low energy string theory to Einstein theory, and at the time of the transition the relevant perturbations were superhorizon size. Most of the observationally relevant scale may exit the horizon during the last moments of the latest inflation stage. Thus, for the validity of the first assumption it is essential that the pre-big bang stage serves during near end of the latest inflation.

Recently, an interesting suggestion was made that the graceful exit problem in the original pre-big bang scenario can be resolved by including the quantum back reaction effect 22]. Since the spacetime curvature term diverges near the pole region $\left(t \simeq t_{0}\right)$, the quantum back reaction could become important as the inflation approaches the pole. In such a case, since the observationally relevant perturbations for the large scale structure leave the horizon most probably during the stringy pre-big bang era, one has to investigate the scalar and the gravitational wave spectrums based on an action which includes higher order string quantum correction terms. Results for observable quantities could be very different, and at the moment, the analysis is beyond the scope of our formulation used in this paper; the pre-big bang scenario considered in this paper does not include effects of the dilaton potential, axion field, and quantum stringy effects like higher order curvature correction terms except for the $f(\phi, R)$ term. [In this case, there remains a possibility that the $n \simeq 4$ and $n_{T} \simeq 3$ power spectrums could be interpreted as the spectrums of the present day superhorizon scale structures which exited the horizon before the stringy quantum era begins.] The general formulation presented in [4, 5, 6, 7, 9] and summarized in $\$$ 河 will be a useful step for extended studies. As a conclusion, although the pre-big bang inflation fails observationally, one cannot blame the string theory on the charge of the faileur. Investigating the structure generation processses in the strong quantum regime is a very important and interesting open problem which is left for future endeavour.

\section{Acknowledgments}

We wish to thank Drs. R. Brustein, M. Gasperini, H. Noh and S.-J. Rey for useful discussions and comments on the manuscript. This work was supported by the Korea Science and Engineering Foundation, Grant No. 95-0702-04-01-3 and through the SRC program of SNU-CTP.

\section{References}

[1] M. Green, J. Schwarz and E. Witten, Superstring Theory, Vol 1 and 2 (Cambridge Univ. Press, Cambridge, 1987).

[2] A. Linde, Particle Physics and Inflationary Cosmology, (Harwood Academic Publishers: New York, 1990); E. W. Kolb and M. S. Turner, The Early Universe, (Addison-Wesley Publishing Company: Seoul, 1994).

[3] G. Veneziano, Phys. Lett. B 265 (1991) 287; M. Gasperini and G. Veneziano, Astroparticle Phys. 1 (1993) 317; For a collection of papers on the pre-big bang string cosmology see a web location 'http://www.to.infn.it/teorici/gasperini/]'.

[4] J. Hwang, Phys. Rev. D 53 (1996) 762.

[5] J. Hwang and H. Noh, Phys. Rev. D 54 (1996) 1460.

[6] J. Hwang, Class. Quant. Grav. 14 (1997) 1981. 
[7] J. Hwang, Class. Quant. Grav. 14 (1997) 3327.

[8] J. Hwang, Astrophys. J., 375 (1991) 443.

[9] J. Hwang, gr-qc/9710061 (1997).

[10] C. G. Callan, D. Friedan, E. J. Martinec and M. J. Perry, Nucl. Phys. B 262 (1985) 593.

[11] J. Hwang, Class. Quantum Grav., 7 (1990) 1613.

[12] L. H. Ford and L. Parker, Phys. Rev. D 16 (1977) 1601.

[13] L. Amendola, M. Litterio and F. Occhionero, Phys. Lett. B 231 (1989) 43; Class. Quantum Grav. 8 (1991) L133; V. F. Mukhanov, H. A. Feldman and R. H. Brandenberger, Physics Reports 215 (1992) 203; J. D. Barrow, J. P. Mimoso and M. R. de G. Maia, Phys. Rev. D 48 (1993) 3630.

[14] R. Brustein, M. Gasperini, M. Giovannini, V. Mukhanov and G. Veneziano, Phys. Rev. D 51 (1995) 6744 .

[15] M. Gasperini and M. Giovannini, Phys. Lett. B 282 (1992) 36; Class. Quant. Grav. 9 (1992) L137; Phys. Rev. D 47 (1993) 1519; R. Brustein, M. Gasperini, M. Giovannini and G. Veneziano, Phys. Lett. B 361 (1995) 45; M. Gasperini, Report No. hep-th/9607146 (unpublished) and references therein.

[16] E. Calzetta and B. L. Hu, Phys. Rev. D 52 (1995) 6770.

[17] J. M. Bardeen, P. J. Steinhardt and M. S. Turner, Phys. Rev. D 28 (1983) 679.

[18] Sec. 3.3.2 and Table 8 of J. Hwang, Astrophys. J. 415 (1993) 486; Sec. 3.8 and Table 1 of J. Hwang, Astrophys. J. 427 (1994) 542.

[19] L. F. Abbott and D. D. Harari, Nucl. Phys. B 264 (1986) 487; B. Allen, Phys. Rev. D 37 (1988) 2078; V. Sahni, Phys. Rev. D 42 (1990) 453.

[20] A. A. Starobinsky, JETP Lett. 30 (1979) 682.

[21] R. Brustein and G. Veneziano, Phys. Lett. B 329 (1994) 429; R. Easther, K. Maeda and D. Wands, Phys. Rev. D 53 (1996) 4247; N. Kaloper, R. Madden and K. A. Olive, Phys. Lett. B 371 (1996) 34; M. Gasperini, J. Maharana and G. Veneziano, Nucl. Phys. B 472 (1996) 349.

[22] S.-J. Rey, Phys. Rev. Lett. 77 (1996) 1929.

[23] R. Sachs and A. Wolfe, Astrophys. J. 147 (1967) 73; G. Dautcourt, Mon. Not. R. Astron. Soc. 144 (1969) 255.

[24] L. F. Abbott and M. B. Wise, Nucl. Phys. B 244 (1984) 541; A. A. Starobinsky, Sov. Astron. Lett. 11 (1985) 133; B. Allen and S. Koranda, Phys. Rev. D 50 (1994) 3713.

[25] E. F. Bunn, A. R. Liddle, and M. White, Phys. Rev. D 54 (1996) R5917.

[26] M. Gasperini and G. Veneziano, Phys. Rev. D 50 (1994) 2519.

[27] M. J. Duff, in Quantum Gravity 2: A Second Oxford Symposium, Eds. C. J. Isham, R. Penrose and D. W. Sciama (Oxford University Press, Oxford, 1981) 81p. 